\title{
Evaluation of Hedgehog Pathway Inhibitors as a Therapeutic Option for Uterine Leiomyosarcoma Using the Xenograft Model
}

\author{
Natalia Garcia ${ }^{1,2} \cdot$ Mara Ulin $^{1,3} \cdot{\text { Mohamed } \text { Ali }^{1,4} \cdot \text { Ayman Al-Hendy }^{5} \cdot \text { Katia Candido Carvalho }}^{2} \cdot$ Qiwei Yang $^{5}$ (D)
}

Received: 18 April 2021 / Accepted: 26 August 2021 / Published online: 12 October 2021

(c) The Author(s) 2021

\begin{abstract}
Uterine leiomyosarcoma (LMS) contributes to a significant proportion of uterine cancer deaths. It is a rare and high-risk gynecological cancer. LMS is challenging to the treatment due to the resistance of several therapies. The activation of the Hedgehog $(\mathrm{HH})$ pathway has been reported in several types of female cancers. Uterine LMS presents an upregulation of the crucial HH signaling pathway members such as SMO and GLI1. Although targeting the HH pathway exhibited a potent inhibitory effect on the phenotype of uterine LMS in vitro, the effect of the HH inhibitors on LMS growth in vivo has not been identified. The present study aimed to assess the effect of Hedgehog pathway inhibitors (SMO-LDE225 and GLI-Gant61) as a therapeutic option in the xenograft model of uterine LMS. The results demonstrated that LDE225 treatment did not show any inhibitory effect on LMS tumor growth; however, treatment with GLI inhibitor (Gant61) induced a remarkable tumor regression with a significant decrease in Ki67 expression, compared to control $(p<0.01)$. Moreover, administration of Gant61 decreased the expression of GLI1, GLI target genes BMP4 and $c-M Y C(p<0.05)$, indicating that the HH pathway is implicated in the LMS experimental model. In conclusion, our studies demonstrate for the first time that GLI inhibitor (Gant61), but not SMO inhibitor (LDE225), shows a potent inhibitory effect on LMS tumor growth and concomitantly suppresses the expression of GLI1- and GLI-targeted genes using the xenograft model of uterine LMS.
\end{abstract}

Keywords Uterine leiomyosarcoma $\cdot$ Hedgehog signaling $\cdot$ SMO inhibitor $\cdot$ GLI inhibitor

\section{Introduction}

Uterine leiomyosarcoma (LMS) contributes to a significant proportion of uterine cancer deaths [1-4]. It is a rare and aggressive gynecological cancer, which accounts for $1 \%$ of all uterine malignancies. LMS is challenging to treatment

Qiwei Yang

yangq@bsd.uchicago.edu

1 Department of Surgery, University of Illinois at Chicago, Chicago, IL, USA

2 Laboratório de Ginecologia Estrutural e Molecular - LIM 58, Disciplina de Ginecologia, Hospital das Clinicas da Faculdade de Medicina da Universidade de Sao Paulo, HCFMUSP, Sao Paulo, Brazil

3 Department of Pathology, University of Illinois at Chicago, Chicago, IL, USA

4 Clinical Pharmacy Department, Faculty of Pharmacy, Ain Shams University, Cairo, Egypt

5 Department of Obstetrics and Gynecology, University of Chicago, Chicago, IL, USA exhibiting resistance to several therapies including FDAapproved drugs such as pazopanib and olaratumab [2, 5-8], evidenced by high rates of recurrence and progression [9, 10]. These characteristics emphasize the need for new therapeutic options for this tumor.

The Hedgehog $(\mathrm{HH})$ pathway activation depends on the HH ligand (SHH, IHH, or DHH) [11]. In the absence of the ligand, the PTCH1 receptor blocks SMO activity. However, when the HH ligand binds to PTCH1, the SMO inhibition is relieved, triggering activation and nuclear translocation of the GLIs transcription factors to regulate the $\mathrm{HH}$ target genes. In the absence of the $\mathrm{HH}$ ligand, the negative regulator SUFU sequesters the GLI proteins in the cytoplasm [12-14].

The deregulation of the HH signaling pathway plays an important role in more than $30 \%$ of human cancers [15]. This deregulation of the $\mathrm{HH}$ pathway contributes to tumor initiation and progression [16-19]. The activation of the HH pathway has been described in several types of female cancer, including uterine LMS [20-27]. In LMS, the HH pathway was described for the first time by Garcia and collaborators 
[25], who showed that the protein expression of SMO and GLI1, the crucial members of the HH signaling pathway, was increased in formalin-fixed paraffin-embedded (FFPE) LMS' patient samples, compared to uterine leiomyoma and myometrium [25].

Due to the important role of key $\mathrm{HH}$ components in cancer progression, targeting SMO and the GLIs have been demonstrated to be a useful strategy to block the $\mathrm{HH}$ signaling pathway activity and suppress the tumor progression [28-33]. SMO and GLI inhibitors have been shown to exert an anti-cancer activity in vitro and in vivo on different types of cancer [20, 26, 34-36]. Furthermore, several SMO inhibitors are approved by the FDA (GDC099, LDE225, and PF-04449913) and have been tested in clinical trials showing promising results in breast cancer [37], basal cell carcinoma [38, 39], medulloblastoma [40], and pancreatic cancer [41].

Recently, our in vitro study demonstrated that LDE225 (SMO) and Gant61 (GLI) inhibitors were capable of blocking the HH pathway signaling with a significant decrease in the LMS cell proliferation and migration with prominent apoptosis enhancement [26]. However, the effects of the $\mathrm{HH}$ inhibitors on LMS growth in vivo are unclear. The present study aimed to assess the effect of HH pathway inhibitors as a therapeutic option using the xenograft model of uterine LMS.

\section{Material and Methods}

\section{Cell Culture and Reagents}

The human uterine LMS cell line (SK-UT1, ATCC® HTB$114^{\mathrm{TM}}$ ) was purchased from the ATCC (Manassas, VA, USA), and it was cultured in the recommended media and growth condition. GLI inhibitor, Gant61, was purchased from Sigma Aldrich (St. Louis, MO, USA) and SMO inhibitor LDE225 from Selleck Chemical (Houston, TX, USA).

\section{Leiomyosarcoma Xenograft Tumors}

Twenty-nine nu/nu nude mice were purchased from Charles River (Wilmington, MA, USA). The mice were handled according to the approved protocol (18-174) and all mice were maintained in a 12-h light/dark cycle and provided with water and standard diet ad libitum in a pathogen-free facility under climate control. $2 \times 10^{7}$ of the human LMS cells were inoculated into the right flank of mice with 1:1 Matrigel (Corning, Corning NY, USA) and fetal bovine serum (FBS) according to the previous publications [42-46]. After the tumor development, the animals were randomized separately into three groups, SMO inhibitor LDE225 $(n=5)$, GLI inhibitor Gant61 $(n=6 \times 2)$, and control $(n=6 \times 2)$. Gli treatments were repeated compared to the vehicle control group to receive enough tissues for cellular and molecular analysis due to Gant61's marked inhibition of tumor growth; $20 \mathrm{mg} / \mathrm{kg}$ of LDE225 [47], $20 \mathrm{mg} / \mathrm{kg}$ of Gant61 [20, 48, 49], or corn oil (vehicle) were administrated via oral gavage three times per week for 10 days to the Gant61 group and 21 days to the LDE225 group. After the treatment, the animals were sacrificed, and tumors were collected for histopathological and RNA and protein expression profile analysis.

\section{RNA Extraction and Gene Expression}

The total RNA was isolated using TRIzol Reagent (Invitrogen, CA, USA). The concentration was determined using NanoDrop (Thermo Scientific, Waltham, MA). The High Capacity cDNA Transcription Kit (Thermo Scientific, Waltham, MA) was used to perform the reverse-transcribed to complementary DNA by one microgram of the total RNA. The real-time PCR was performed using the CFX96 PCR instrument using SYBR Green Supermix (Bio-Rad, Hercules, CA, USA). The results are presented as relative gene expression using CFX Maestro ${ }^{\mathrm{TM}}$. The primers for detecting the gene expression profile are listed in Table 1.

\section{Morphological (H\&E) and Immunohistochemistry (IHC) Assessment}

The LMS tumors were fixed in $10 \%$ buffered formalin for $24 \mathrm{~h}$, then embedded with paraffin and subjected to H\&E and IHC staining by Research Histology and Tissue Imaging Core at the University of Illinois at Chicago. The antibodies used in this study are shown in Table 2. The IHC analysis was performed using a semi-quantitative score considering the percentage of labeled cells $(0$, negative; $1,<10 \%$ of the cells; $2,10-50 \%$ of the cells; $3,50-75 \%$ of the cells; $4,>75 \%$ of the cells) and the intensity of the immunostaining ( 0 , no staining; 1 , weak; 2 , mild; 3 , strong staining). The multiplication of both scores resulted in a final quotient ranging from 0 to 12 [25]. The slides were scanned using the Aperio image scope software (Aperio Technologies, Inc., Vista, VA, USA).

\section{Statistical Analysis}

The data are expressed as the means \pm standard error by using the GraphPad Prism 5 software. Statistical analyses were carried out using the parametric (Student's $t$-test or analysis of variance ANOVA followed by Tukey posttest) or nonparametric distribution (Mann-Whitney test or Kruskal-Wallis followed by Dunns post-test). The significant difference was defined as $p<0.05$. 
Table 1 qRT-PCR primers sequences

\begin{tabular}{lll}
\hline Gene/symbol & Forward sequence & Reverse sequence \\
\hline$G L I 1$ & AGCCTTCAGCAATGCCAGTGAC & GTCAGGACCATGCACTGTCTTG \\
$G L I 2$ & CTGTGGGTTAGGGATGGACTG & GTAAAGTGGGTGGACGTTGCA \\
$G L I 3$ & GTGCTCCACTCGAACAGA & TCCAGGACTTTCATCCTCATTAGA \\
$S M O$ & TGAAGGCTGCACGAATGAGG & CTTGGGGTTGTCTGTCCGAA \\
$B C L-2$ & TGTGTGTGGAGAGCGTCAAC & GCCAGAGAAATCAAACAGAGG \\
$C C N D 1$ & CTTCAAATGTGTGCAGAAGG & CTCGCACTTCTGTTCCTC \\
$P 21$ & CCCTTGTCCTTTCCCTTCAGTAC & GTGGGACAGGCACCTCAGA \\
$B M P 4$ & CGTAGCCCTAAGCATCACTCACA & GCGCCGGCAGTTCTTATTCT \\
$F o x M 1$ & GGGCGCACGGCGGAAGATGAA & CCACTCTTCCAAGGGAGGGCTC \\
$c-M Y C$ & AATGAAAAGGCCCCCAAGGTAGTTATCC & GTCGTTTCCGCAACAAGTCCTCTTC \\
$P 27$ & ATGTCAAACGTGCGAGTGTCT & TTACGTTTGACGTCTTCTGA \\
$V E G F$ & CTACCTCCACCATGCCAAGT & GCAGTAGCTGCGCTGATAGA \\
$T P 53$ & TGTAGTGGATGGTGGTACAG & CGTGTGGAGTATTTGGATGAC \\
$B 2 M$ & CAGCCCAAGATAGTTAAGTG & CCCTCCTAGAGCTACCTGT \\
\hline
\end{tabular}

Table 2 Description and details of antibodies used in this study

\begin{tabular}{lllll}
\hline Antibody & Manufacturer & $\begin{array}{l}\text { Species, monoclonal, or } \\
\text { polyclonal }\end{array}$ & $\begin{array}{l}\text { Application and } \\
\text { dilution }\end{array}$ & Catalog number \\
\hline SMO & GeneTex & Rabbit, polyclonal & IHC, 1:400 & GTX02530 \\
GLI1 & Sigma & Rabbit, polyclonal & IHC, 1:200 & ABC217 \\
KI67 & Abcam & Rabbit, monoclonal & IHC, 1:200 & ab16667 \\
\hline
\end{tabular}

\section{Results}

\section{Inhibition of Hedgehog Pathway Promotes Tumor Regression in the Xenograft Model of Uterine LMS}

The HH inhibitors, SMO-LDE225 and GLI- Gant61, were selected based on our previous findings that these two HH inhibitors exhibited a significant inhibitory effect (decreasing cell proliferation, migration, and increasing apoptosis index) on LMS cells in vitro [26]. In this study, after the tumors developed, the animals were randomly separated into three groups (SMO inhibitor, GLI inhibitor, and control). SMO inhibitor group was treated for 21 days with $20 \mathrm{mg} / \mathrm{kg}$ of LDE225. In contrast to the in vitro inhibitory effect, the LDE225 treatment did not show any inhibitory effect on LMS tumor growth. In addition, no significant difference in tumor volume between the LDE225 treatment and the control group was observed (Fig. 1A, B). However, the animals treated with $20 \mathrm{mg} / \mathrm{kg}$ of Gant61, GLI inhibitor, for 10 days showed a significant tumor regression compared to control $(p<0.01)$ (Fig. 1A, B). Both treatments were well tolerated by the animals, without causing weight loss or other side effects in the mice behavior (Fig. 1C).

\section{Targeting Hedgehog Pathway Decreases HH Activity and Proliferation in the Xenograft Model of Uterine LMS}

After the treatment with the HH inhibitors, LDE225 and Gant61, the tumors were collected, and H\&E and IHC analyses were performed to evaluate the expression of proliferation marker (Ki67) and HH components (SMO or GLI1). The tumors treated with LDE225, SMO inhibitor, exhibited no change in the expression of SMO and Ki67 compared to the controls (Fig. 2A, B). However, the tumors treated with GLI inhibitor (Gant61) showed a significant decrease in GLI1 and Ki67 protein expression compared to the control $(p<0.0001)$ (Fig. 3A, B).

\section{Targeting Hedgehog Pathway Decreases Gene Expression of HH Components in Xenograft Model of Uterine LMS}

After 21 days of the treatment with SMO inhibitor (LDE225), the tumors did not show a difference in the gene expression profile of $S M O, G L I 1$, or GLI2 when compared to the control group (Fig. 4A). The tumors treated for 10 days with GLI inhibitor (Gant61) showed a decreased expression 
A

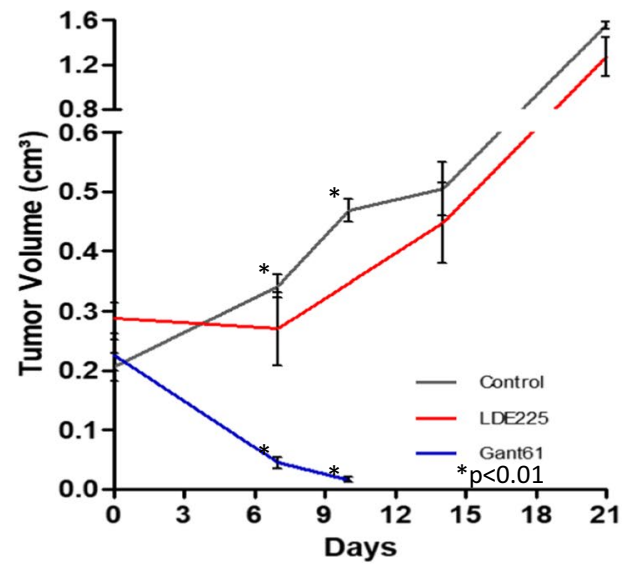

B
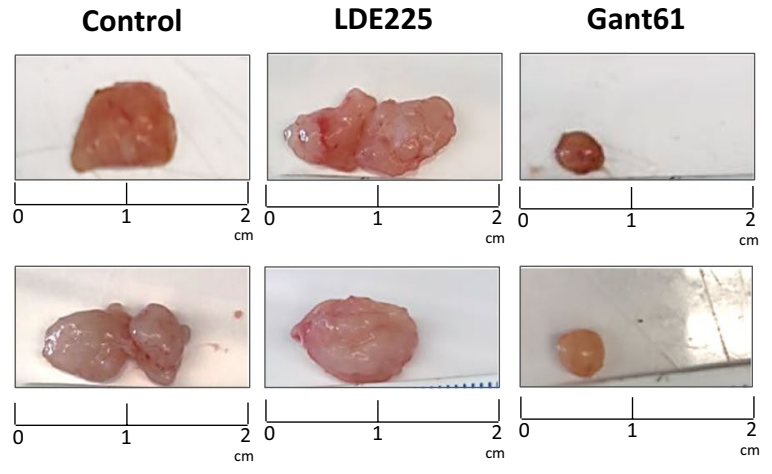

C

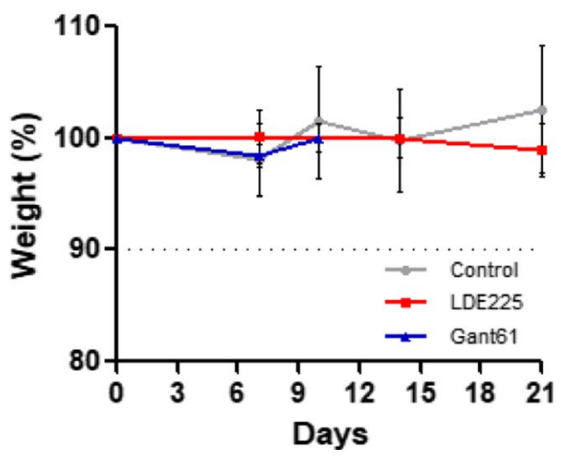

Fig. 1 Response curve of LMS tumors treated with SMO (LDE225 $20 \mathrm{mg} / \mathrm{kg}$ ) or GLI inhibitors (Gant61 $20 \mathrm{mg} / \mathrm{kg}$ ). A The animals bearing tumors were administered with $20 \mathrm{mg} / \mathrm{kg}$ of LDE225 (SMO inhibitor) or Gant61 (GLI inhibitor), and the tumor growth was measured during the treatment. The relative tumor volume was calculated and plotted. B Pictures represented the tumor volume at the end of the treatment with SMO or GLI inhibitors compared to control. C Graph summarizing the percent body weight of mice during the treatment with SMO or GLI inhibitor

of $G L I 1$ compared to control $(p<0.05)$. Gant61 treatment did not alter the expression of GLI2 and GLI3 in LMS tumors (Fig. 4B). GLI-targeted genes (BCL-2, CCND1, $P 21, B M P 4, F O X M 1, C-M Y C, P 27, V E G F$, and TP53) were selected to assess their expression after the Gant61 treatment. The expression of $B M P 4$ and $c-M Y C$ was significantly decreased in the Gant61-treated tumors compared to the control group $(p<0.05)$. The expression of other GLItargeted genes (BCL-2, CCND1, P21, FOXM1, VEGF, and TP53) did not show a significant difference after treatment with Gant61 (Fig. 4C).

\section{Discussion}

To the best of our knowledge, this study was the first to assess the effect of the SMO and GLI inhibitors on LMS growth in the xenograft model and determined if both inhibitors were capable of suppressing the activation of the $\mathrm{HH}$ signaling pathway in LMS in vivo. We have demonstrated previously that the HH signaling pathway is deregulated in LMS [25, 26]. Our key finding was the upregulation of SMO and the transcription factor GLI1 in LMS cell line compared to normal uterine myometrium cells [25] and human samples [26]. Moreover, inhibition of the HH pathway using SMO and GLI inhibitors suppressed the LMS proliferation and migration and increased LMS apoptosis rate in vitro.

Based on our previous results, the use of the LMS xenograft model to confirm the efficacy of SMO and GLI inhibitors was needed to understand the deregulation of HH pathway in LMS, as well as therapeutic options for this aggressive cancer. In this study, the treatment with LDE225, the SMO inhibitor, showed an inefficient suppression of LMS tumor growth, concomitantly with no changes in SMO and Ki67 protein expression, as well as in gene expression of SMO, GLII, and GLI2.

Several studies demonstrated that LDE225 as an SMO inhibitor exhibited an anti-cancer efficacy in different types of tumors via blocking the HH pathway activity. In an animal model with glioblastoma, the treatment with LDE225 decreased the tumor size with downregulation of GLI1, GLI2, PTCH1, and SMO [50]. In a pancreatic tumor, LDE225 blocked the activation of the $\mathrm{HH}$ signaling pathway, decreasing GLI1 and PTCH1 gene expression (51]. In melanoma, LDE225 decreases the tumor size with a decrease of GLI1 expression [52].

Our results showed that the administration of SMO inhibitor (LDE225) in the animal model of LMS was inefficient to suppress the tumor growth. This result is inconsistent with our previous report showing that LDE225 treatment revealed an efficiency to block the HH pathway activity, inducing a significant decrease in cell migration, proliferation, and increased apoptosis rate in LMS cells in vitro [26].

Studies comparing in vitro and in vivo experiments have shown to be complex due to the difficulty of reproducing the tumor and non-tumor cell interactions [53]. Cancer treatment has been challenging because of the complexity and heterogeneity of the tumor $[54,55]$. Tumor 
Fig. $2 \mathrm{H} \& \mathrm{E}$ and immunohistochemistry staining of SK-UT1 xenograft tumors. A Immunohistochemical staining for SMO and $\mathrm{Ki} 67$ and hematoxylin and eosin (H\&E) staining of tumors treated with SMO inhibitor (LDE225) for 21 days. B Graph summarizing the immunohistochemical staining score (intensity $\mathrm{x}$ frequency) for SMO and $\mathrm{Ki} 67$
A

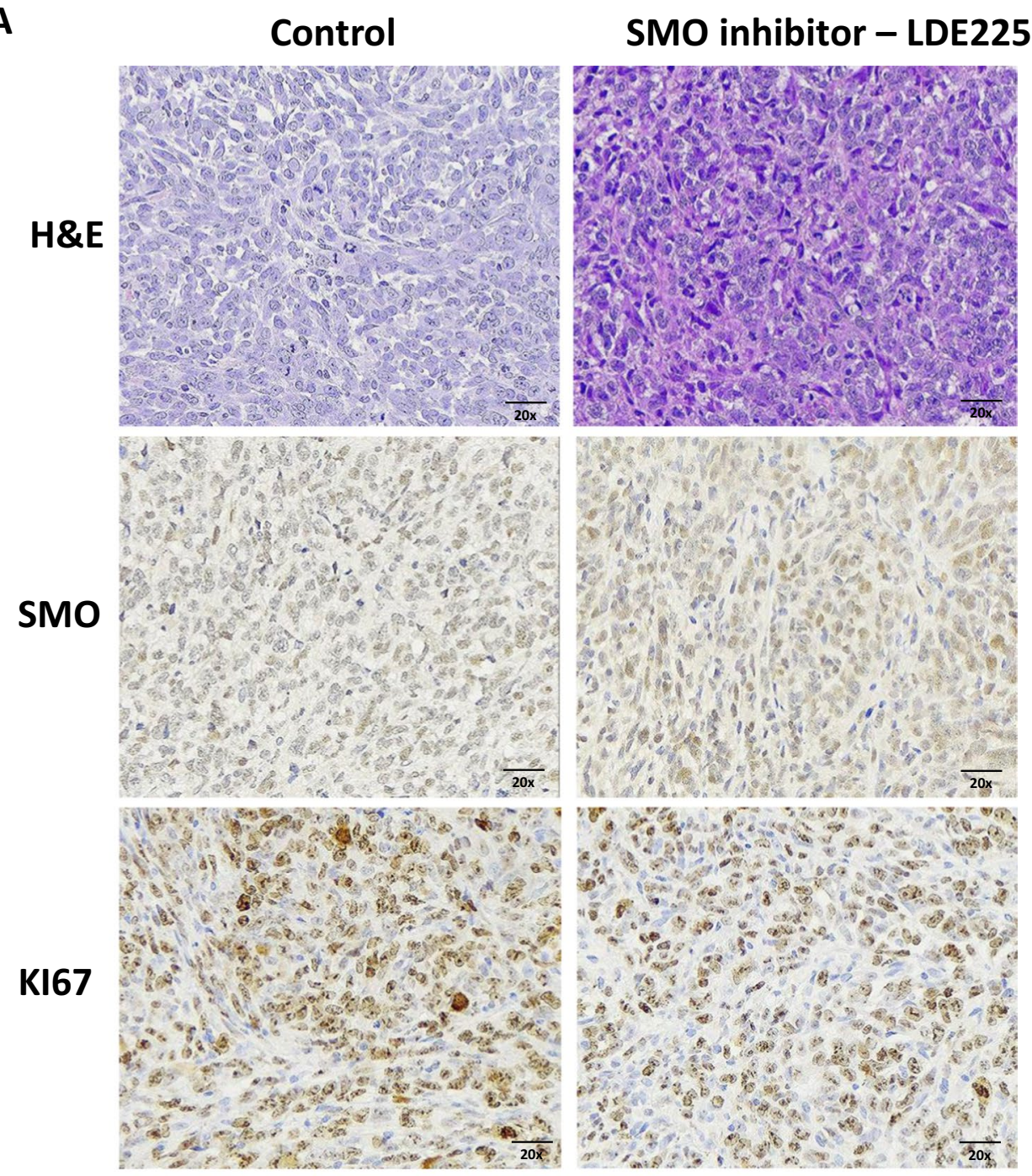

B

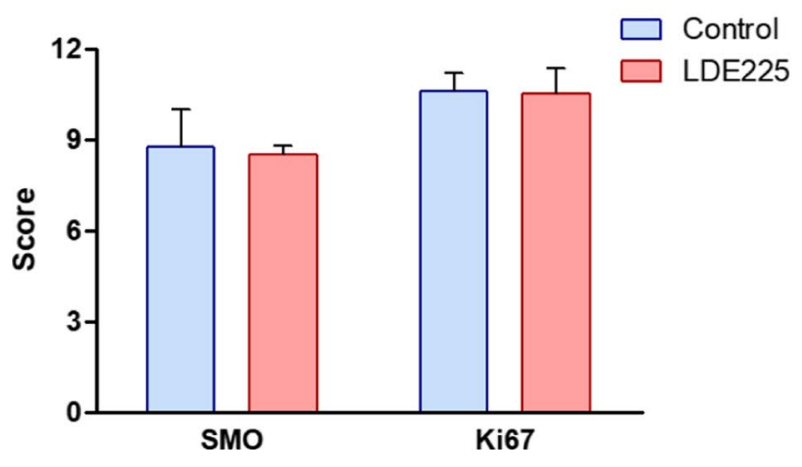

progression is profoundly influenced by the interactions of cancer cells with their environment. The tumor microenvironment consists of different non-cancer cell types and their stroma, which have a role in the structure, physiology, and function of the tumor [56]. In vitro experiments have been helpful to understand cell behavior, but they have cell bioactivities different from the in vivo response.
The animal models mimic these complex interactions with their surrounding cells [53].

Gant61 as a GLI inhibitor has been used as a novel anti-cancer drug in preclinical studies [57]. In our previous studies, we demonstrated that GLI1 exhibited a higher expression in LMS compared to the myometrium and benign leiomyoma [25]. We also showed that the treatment with 
Fig. 3 Histology and immunohistochemical analysis of the SK-UT1 xenograft tumors treated with GLI inhibitor. A Immunohistochemical staining for GLI1 and Ki67 and hematoxylin and eosin (H\&E) staining of tumors treated with GLI inhibitor (Gant61) for 10 days. B Graph summarizing the immunohistochemical staining score (intensity $\times$ frequency) for GLI1 and Ki67. $p<0.0001$
A

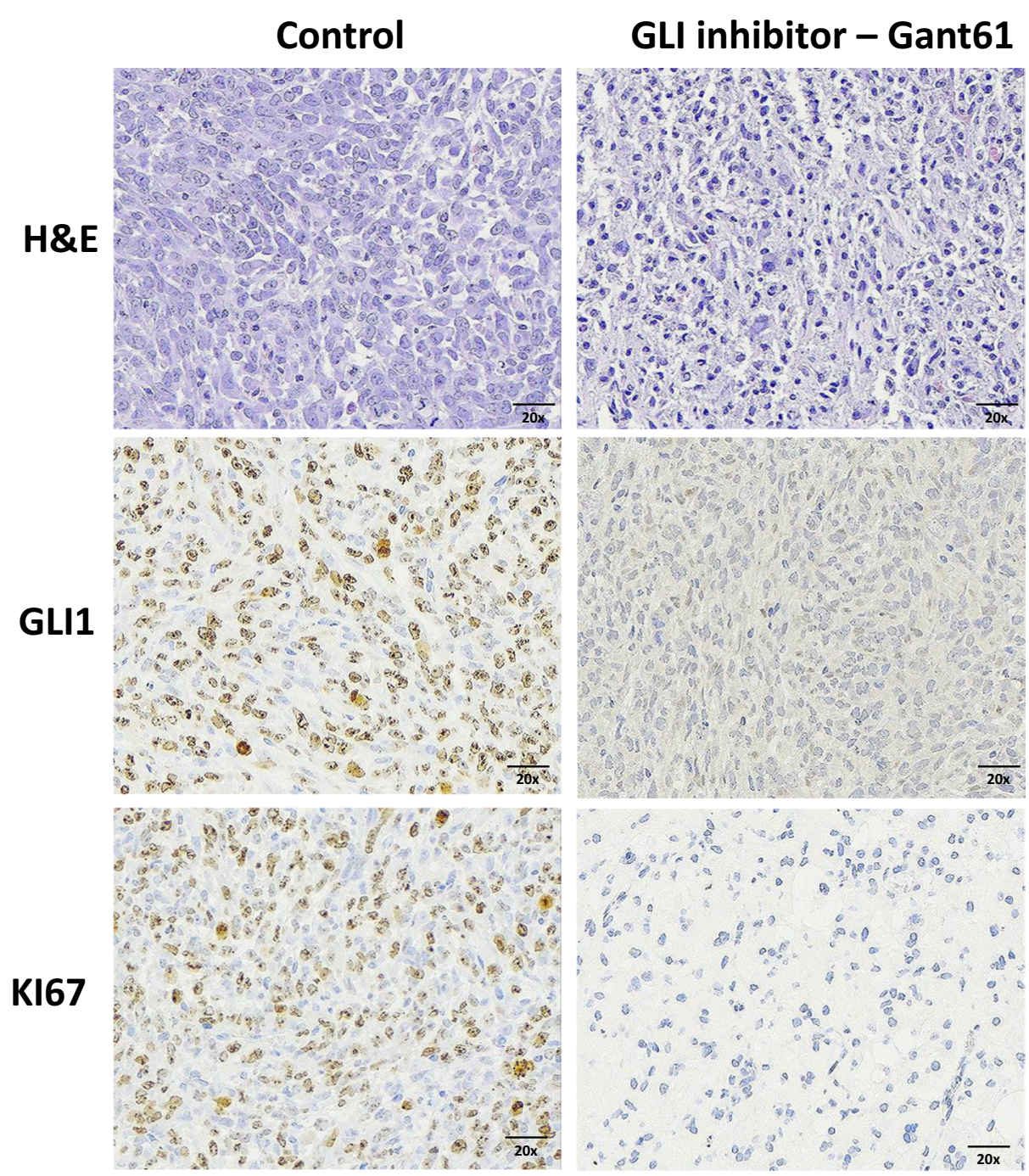

B

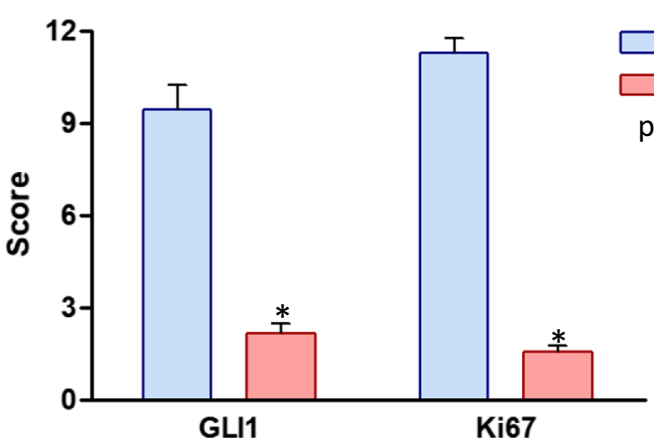

Control

Gant61 $p<0.0001$
Gant61 blocked the GLI1 expression, decreasing cell proliferation and migration and increasing apoptosis index in LMS cells [26]. In this study, we tested the efficiency of Gant61 used as a therapeutic option to treat uterine LMS in the xenograft model, and our results showed a remarkable regression of the LMS growth with decreased expression of Gli and GLI-target genes BMP4 and c-MYC. The use of
Gant61 as a treatment option to LMS may have a potential promising outcome compared to other agents to overcome drug resistance. Recently, Nakae et al. [46] showed that CD70 antibody conjugate was able to inhibit the LMS tumor growth in a PDX model. This model possesses several advantages including preserving tumor heterogeneity and lineage hierarchy, allowing for effective chronological tumor 
Fig. 4 Gene expression analysis of LMS tumors treated with SMO or GLI inhibitors. A Relative quantification of the expression of $S M O, G L I 1$, and GLI2 in the LMS tumors after 21 days of treatment with LDE225 or vehicle (corn oil). B Relative quantification of the GLII, GLI2, and GLI3 in the LMS tumors after 10 days of treatment with Gant 61 or vehicle (corn oil). C RNA expression of GLI-target genes $(B C L-2$, CCND1, P21, BMP4, FOXM1, $C-M Y C, P 27, V E G F$, and TP53) in the LMS tumors after 10 days of treatment with Gant61 or vehicle (corn oil). $p<0.05$
A

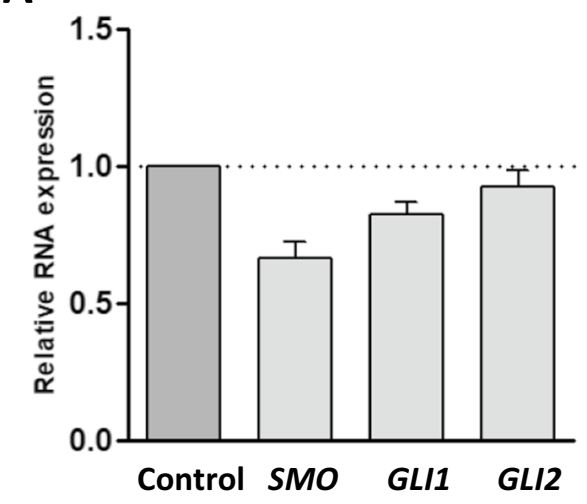

B

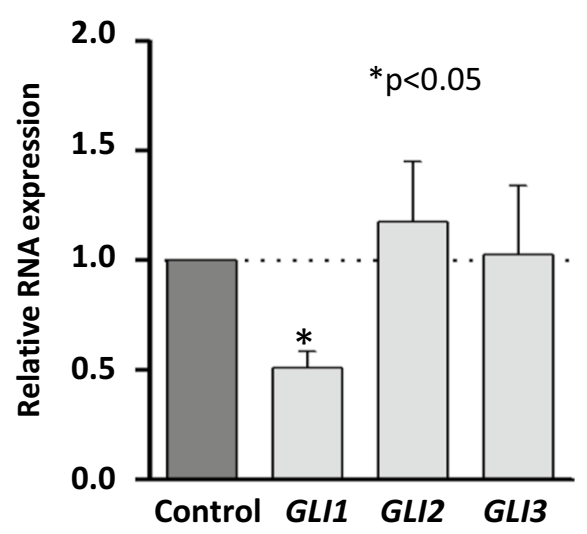

C

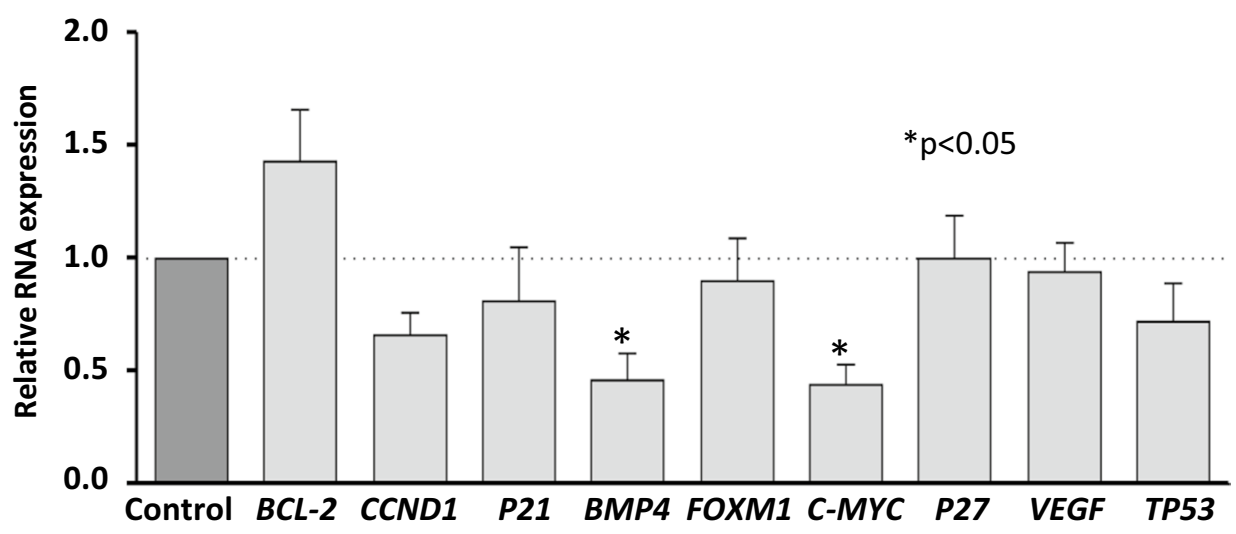

size monitoring, and potential applications in personalized medical treatments. In this regard, evaluating of Gli inhibitor in the PDX model is warranted.

The use of Gant61 to block the HH pathway has been described in other types of tumor. For instance, in breast cancer, Gant61 decreases tumor growth [20]. In thyroid tumors, the treatment downregulated the GLI1 protein expression and reduced the tumor volume [48]. In a xenograft model of osteosarcoma, Gant61 promoted tumor regression (49). $c-M Y C$ and BMP4 have been described as GLI target genes $[58,59]$. In medulloblastoma, treatment with SMO inhibitor,
GDC0449, showed a decrease in the c-MYC RNA expression and protein levels [59]. We previously described the upregulation of BMP4 in FFPE samples in LMS [25], and in human colon carcinoma, BMP4 expression was increased after stimulation with $\mathrm{HH}$ agonists [58].

In conclusion, our studies demonstrated for the first time, to the best of our knowledge, that GLI inhibitor (Gant61), but not SMO inhibitor (LDE225), showed a potent inhibitory effect on LMS tumor growth and concomitantly suppressed the expression of GLI1 and GLI-targeted genes in the xenograft model of uterine LMS. Our studies suggest 
that Gant61 targeting HH pathway might be considered a promising therapeutic option to inhibit the LMS progression.

Author Contribution NG designed and conducted the experiments, data acquisition, and manuscript preparation. MU conducted the experiments and manuscript preparation. MA conducted the experiment. $\mathrm{KCC}, \mathrm{AA}$, and QY contributed to the conceptual design and manuscript revision.

Funding This study was supported in part by Fundação de Amparo à Pesquisa do Estado de São Paulo (FAPESP) (FAPESP 2017/24448-1) for Natalia Garcia's scholarship and National Institutes of Health (NIH) grants RO1 HD094378; RO1 ES028615; U54 MD007602 and RO1 HD094380.

Data Availability All data generated or analyzed during this study are included in this published article.

Code Availability Not applicable.

\section{Declarations}

Ethics Approval All applicable international, national, and/or institutional guidelines for the care and use of animals were followed, and IACUC approval of the University of Illinois at Chicago was taken.

Consent to Participate This article does not contain any studies with human participants performed by any of the authors.

Consent for Publication All authors consented with the publication.

Competing Interests Dr. Ayman Al-Hendy is consulting Abbvie, Bayer, Allergan, and MD Stem Cells for research support. The other authors declare no competing interests.

Open Access This article is licensed under a Creative Commons Attribution 4.0 International License, which permits use, sharing, adaptation, distribution and reproduction in any medium or format, as long as you give appropriate credit to the original author(s) and the source, provide a link to the Creative Commons licence, and indicate if changes were made. The images or other third party material in this article are included in the article's Creative Commons licence, unless indicated otherwise in a credit line to the material. If material is not included in the article's Creative Commons licence and your intended use is not permitted by statutory regulation or exceeds the permitted use, you will need to obtain permission directly from the copyright holder. To view a copy of this licence, visit http://creativecommons.org/licenses/by/4.0/.

\section{References}

1. Cui RR, Wright JD, Hou JY. Uterine leiomyosarcoma: a review of recent advances in molecular biology, clinical management and outcome. BJOG. 2017;124(7):1028-37.

2. D’Angelo E, Prat J. Uterine sarcomas: a review. Gynecol Oncol. 2010;116(1):131-9.

3. Amant F, Coosemans A, Debiec-Rychter M, Timmerman D, Vergote I. Clinical management of uterine sarcomas. Lancet Oncol. 2009;10(12):1188-98.

4. Giuntoli Ii RL, Metzinger DS, DiMarco CS, Cha SS, Sloan JA, Keeney GL, et al. Retrospective review of 208 patients with leiomyosarcoma of the uterus: prognostic indicators, surgical management, and adjuvant therapy it. Gynecol Oncol. 2003;89(3):460-9.

5. Matsuzaki S, Chang EJ, Yasukawa M, Roman LD, Matsuo K. Surgical and oncologic outcomes of hyperthermic intraperitoneal chemotherapy for uterine leiomyosarcoma: a systematic review of literature. Gynecol Oncol. 2021;161(1):70-7.

6. Libertini M, Hallin M, Thway K, Noujaim J, Benson C, van der Graaf W, et al. Gynecological sarcomas: molecular characteristics, behavior, and histology-driven therapy. Int J Surg Pathol. 2021;29(1):4-20.

7. Rizzo A, Nannini M, Astolfi A, Indio V, De Iaco P, Perrone AM, et al. Impact of chemotherapy in the adjuvant setting of early stage uterine leiomyosarcoma: a systematic review and updated metaanalysis. Cancers (Basel). 2020;12(7):1899.

8. van der Graaf WT, Blay JY, Chawla SP, Kim DW, Bui-Nguyen B, Casali PG, et al. Pazopanib for metastatic soft-tissue sarcoma (PALETTE): a randomised, double-blind, placebo-controlled phase 3 trial. Lancet. 2012;379(9829):1879-86.

9. Hosh M, Antar S, Nazzal A, Warda M, Gibreel A, Refky B. Uterine sarcoma: analysis of 13,089 cases based on surveillance, epidemiology, and end results database. Int J Gynecol Cancer. 2016;26(6):1098-104.

10. Mbatani N, Olawaiye AB, Prat J. Uterine sarcomas. Int J Gynaecol Obstet. 2018;143(Suppl 2):51-8.

11. Gorojankina T. Hedgehog signaling pathway: a novel model and molecular mechanisms of signal transduction. Cell Mol Life Sci. 2016;73(7):1317-32.

12. Amakye D, Jagani Z, Dorsch M. Unraveling the therapeutic potential of the Hedgehog pathway in cancer. Nat Med. 2013;19(11):1410-22.

13 Briscoe J, Therond PP. The mechanisms of Hedgehog signalling and its roles in development and disease. Nat Rev Mol Cell Biol. 2013;14(7):416-29.

14 Choudhry Z, Rikani AA, Choudhry AM, Tariq S, Zakaria F, Asghar MW, et al. Sonic hedgehog signalling pathway: a complex network. Ann Neurosci. 2014;21:28-31.

15. Szkandera J, Kiesslich T, Haybaeck J, Gerger A, Pichler M. Hedgehog signaling pathway in ovarian cancer. Int J Mol Sci. 2013;14(1):1179-96.

16. Tao Y, Mao J, Zhang Q, Li L. Overexpression of Hedgehog signaling molecules and its involvement in triple-negative breast cancer. Oncol Lett. 2011;2(5):995-1001.

17. Noman AS, Uddin M, Rahman MZ, Nayeem MJ, Alam SS, Khatun Z, et al. Overexpression of sonic hedgehog in the triple negative breast cancer: clinicopathological characteristics of high burden breast cancer patients from Bangladesh. Sci Rep. 2016;6:18830.

18. Islam SS, Mokhtari RB, Noman AS, Uddin M, Rahman MZ, Azadi MA, et al. Sonic hedgehog (Shh) signaling promotes tumorigenicity and stemness via activation of epithelial-to-mesenchymal transition (EMT) in bladder cancer. Mol Carcinog. 2016;55(5):537-51.

19. Wang LH, Choi YL, Hua XY, Shin YK, Song YJ, Youn SJ, et al. Increased expression of sonic hedgehog and altered methylation of its promoter region in gastric cancer and its related lesions. Mod Pathol. 2006;19(5):675-83.

20. Benvenuto M, Masuelli L, De Smaele E, Fantini M, Mattera R, Cucchi $\mathrm{D}$, et al. In vitro and in vivo inhibition of breast cancer cell growth by targeting the Hedgehog/GLI pathway with SMO (GDC-0449) or GLI (GANT-61) inhibitors. Oncotarget. 2016;7(8):9250-70.

21. Habib JG, O'Shaughnessy JA. The hedgehog pathway in triplenegative breast cancer. Cancer Med. 2016;5(10):2989-3006.

22. Chen Q, Xu R, Zeng C, Lu Q, Huang D, Shi C, et al. Downregulation of Gli transcription factor leads to the inhibition 
of migration and invasion of ovarian cancer cells via integrin beta4-mediated FAK signaling. PLoS One. 2014;9(2):e88386.

23. Inoue K, Tsubamoto H, Sakata K, Sakane R, Hao H, Hirota $\mathrm{S}$, et al. Expression of Hedgehog signals and growth inhibition by itraconazole in endometrial cancer. Anticancer Res. 2016;36(1):149-53.

24. Sharma A, De R, Javed S, Srinivasan R, Pal A, Bhattacharyya $\mathrm{S}$. Sonic hedgehog pathway activation regulates cervical cancer stem cell characteristics during epithelial to mesenchymal transition. J Cell Physiol. 2019;1-16.

25. Garcia N, Bozzini N, Baiocchi G, da Cunha IW, Maciel GA, Soares JMJ, et al. May Sonic Hedgehog proteins be markers for malignancy in uterine smooth muscle tumors? Hum Pathol. 2016;50:43-50.

26. Garcia N, Al-Hendy A, Baracat EC, Carvalho KC, Yang Q. Targeting hedgehog pathway and DNA methyltransferases in uterine leiomyosarcoma cells. Cells. 2020;10(1):53.

27. Garcia N, Ulin M, Al-Hendy A, Yang Q. The role of Hedgehog pathway in female cancers. J Cancer Sci Clin Ther. 2020;4(4):487-98.

28. Chen JK, Taipale J, Cooper MK, Beachy PA. Inhibition of Hedgehog signaling by direct binding of cyclopamine to Smoothened. Genes Dev. 2002;16(21):2743-8.

29. Burness CB, Scott LJ. Sonidegib: a review in locally advanced basal cell carcinoma. Target Oncol. 2016;11(2):239-46.

30. D'Amato C, Rosa R, Marciano R, D'Amato V, Formisano L, Nappi L, et al. Inhibition of Hedgehog signalling by NVP-LDE225 (Erismodegib) interferes with growth and invasion of human renal cell carcinoma cells. Br J Cancer. 2014;111(6):1168-79.

31. Gonnissen A, Isebaert S, McKee CM, Dok R, Haustermans K, Muschel RJ. The hedgehog inhibitor GANT61 sensitizes prostate cancer cells to ionizing radiation both in vitro and in vivo. Oncotarget. 2016;7(51):84286-98.

32. Wickstrom M, Dyberg C, Shimokawa T, Milosevic J, Baryawno N, Fuskevag OM, et al. Targeting the hedgehog signal transduction pathway at the level of GLI inhibits neuroblastoma cell growth in vitro and in vivo. Int J Cancer. 2013;132(7):1516-24.

33. Carpenter RL, Ray H. Safety and tolerability of Sonic Hedgehog pathway inhibitors in cancer. Drug Saf. 2019;42(2):263-79.

34. Kawabata N, Ijiri K, Ishidou Y, Yamamoto T, Nagao H, Nagano $\mathrm{S}$, et al. Pharmacological inhibition of the Hedgehog pathway prevents human rhabdomyosarcoma cell growth. Int J Oncol. 2011;39(4):899-906.

35. Huang L, Walter V, Hayes DN, Onaitis M. Hedgehog-GLI signaling inhibition suppresses tumor growth in squamous lung cancer. Clin Cancer Res. 2014;20(6):1566-75.

36. Tong W, Qiu L, Qi M, Liu J, Hu K, Lin W, et al. GANT61 and GDC-0449 induce apoptosis of prostate cancer stem cells through a GLI-dependent mechanism. J Cell Biochem. 2018;119(4):3641-52.

37. Ruiz-Borrego M, Jimenez B, Antolín S, García-Saenz JA, Corral J, Jerez Y, et al. A phase Ib study of sonidegib (LDE225), an oral small molecule inhibitor of smoothened or Hedgehog pathway, in combination with docetaxel in triple negative advanced breast cancer patients: GEICAM/2012-12 (EDALINE) study. Invest New Drugs. 2019;37(1):98-108.

38. Bendell J, Andre V, Ho A, Kudchadkar R, Migden M, Infante J, et al. Phase I study of LY2940680, a Smo antagonist, in patients with advanced cancer including treatment-naïve and previously treated basal cell carcinoma. Clin Cancer Res. 2018;24(9):2082-91.

39. Danial C, Sarin KY, Oro AE, Chang AL. An investigator-initiated open-label trial of sonidegib in advanced basal cell carcinoma patients resistant to vismodegib. Clin Cancer Res. 2016;22(6):1325-9.
40. Kieran MW, Chisholm J, Casanova M, Brandes AA, Aerts I, Bouffet E, et al. Phase I study of oral sonidegib (LDE225) in pediatric brain and solid tumors and a phase II study in children and adults with relapsed medulloblastoma. Neuro Oncol. 2017;19(11):1542-52.

41. Catenacci DV, Junttila MR, Karrison T, Bahary N, Horiba MN, Nattam SR, et al. Randomized phase Ib/II study of gemcitabine plus placebo or vismodegib, a Hedgehog pathway inhibitor, in patients with metastatic pancreatic cancer. J Clin Oncol. 2015;33(36):4284-92.

42. Robles AJ, Kurmasheva RT, Bandyopadhyay A, Phelps DA, Erickson SW, Lai Z, et al. Evaluation of eribulin combined with irinotecan for treatment of pediatric cancer xenografts. Clin Cancer Res. 2020;26(12):3012-23.

43. Fontaine SD, Ashley GW, Houghton PJ, Kurmasheva RT, Diolaiti $\mathrm{M}$, Ashworth A, et al. A very long-acting PARP inhibitor suppresses cancer cell growth in DNA repair-deficient tumor models. Cancer Res. 2021;81(4):1076-86.

44. Xia L, Bouamar H, Gu X, Zeballos C, Qin T, Wang B, et al. Gli2 mediates the development of castration-resistant prostate cancer. Int J Oncol. 2020;57(1):100-12.

45. Ghilu S, Li Q, Fontaine SD, Santi DV, Kurmasheva RT, Zheng $\mathrm{S}$, et al. Prospective use of the single-mouse experimental design for the evaluation of PLX038A. Cancer Chemother Pharmacol. 2020;85(2):251-63.

46. Nakae R, Matsuzaki S, Serada S, Matsuo K, Shiomi M, Sato $\mathrm{K}$, et al. CD70 antibody-drug conjugate as a potential therapeutic agent for uterine leiomyosarcoma. Am J Obstet Gynecol. 2021;224(2):197.e1-197.e23.

47. Della Corte CM, Bellevicine C, Vicidomini G, Vitagliano D, Malapelle U, Accardo M, et al. SMO gene amplification and activation of the Hedgehog pathway as novel mechanisms of resistance to anti-epidermal growth factor receptor drugs in human lung cancer. Clin Cancer Res. 2015;21(20):4686-97.

48. Lu Y, Zhu Y, Deng S, Chen Y, Li W, Sun J, Xu X. Targeting the sonic hedgehog pathway to suppress the expression of the cancer stem cell (CSC)-related transcription factors and CSC-driven thyroid tumor growth. Cancers (Basel). 2021;13(3):418.

49. Zhang KQ, Chu XD. GANT61 plays antitumor effects by inducing oxidative stress through the miRNA-1286/RAB31 axis in osteosarcoma. Cell Biol Int. 2021;45(1):61-73.

50. Nanta R, Shrivastava A, Sharma J, Shankar S, Srivastava RK. Inhibition of sonic hedgehog and PI3K/Akt/mTOR pathways cooperate in suppressing survival, self-renewal and tumorigenic potential of glioblastoma-initiating cells. Mol Cell Biochem. 2019;454(1-2):11-23.

51. Fendrich V, Wiese D, Waldmann J, Lauth M, Heverhagen AE, Rehm J, et al. Hedgehog inhibition with the orally bioavailable Smo antagonist LDE225 represses tumor growth and prolongs survival in a transgenic mouse model of islet cell neoplasms. Ann Surg. 2011;254(5):818-23 discussion 23.

52. Jalili A, Mertz KD, Romanov J, Wagner C, Kalthoff F, Stuetz A, et al. NVP-LDE225, a potent and selective SMOOTHENED antagonist reduces melanoma growth in vitro and in vivo. PLoS One. 2013;8(7):e69064.

53. Hoarau-Véchot J, Rafii A, Touboul C, Pasquier J. Halfway between 2D and Animal Models: Are 3D Cultures the Ideal Tool to Study Cancer-Microenvironment Interactions? Int J Mol Sci. 2018;19(1):181.

54. McMillin DW, Negri JM, Mitsiades CS. The role of tumourstromal interactions in modifying drug response: challenges and opportunities. Nat Rev Drug Discov. 2013;12(3):217-28.

55. Pietras K, Ostman A. Hallmarks of cancer: interactions with the tumor stroma. Exp Cell Res. 2010;316(8):1324-31.

56. Tlsty TD, Coussens LM. Tumor stroma and regulation of cancer development. Annu Rev Pathol. 2006;1:119-50. 
57 Peer E, Tesanovic S, Aberger F. Next-generation Hedgehog/ GLI pathway inhibitors for cancer therapy. Cancers (Basel). 2019;11(4):538.

58. Yoshimoto AN, Bernardazzi C, Carneiro AJ, Elia CC, Martinusso CA, Ventura GM, et al. Hedgehog pathway signaling regulates human colon carcinoma HT-29 epithelial cell line apoptosis and cytokine secretion. PLoS One. 2012;7(9):e45332.
59. Chaturvedi NK, Kling MJ, Coulter DW, McGuire TR, Ray S, Kesherwani $\mathrm{V}$, et al. Improved therapy for medulloblastoma: targeting hedgehog and PI3K-mTOR signaling pathways in combination with chemotherapy. Oncotarget. 2018;9(24):16619-33. 